\title{
Virtual Modeling of a Cleft Lip and Palate Patient
}

\author{
İ́fan Karadede1, Özkan Adıgüzel ${ }^{2}$, Törün Özer ${ }^{3}$, Fundagül Bilgiç ${ }^{4}$, Renin Özhal İzol ${ }^{5}$ \\ ${ }^{1}$ Professor, Dicle University, Faculty of Dentistry, Department of Orthodontics, Diyarbakır, TURKEY \\ ${ }^{2}$ Assoc. Professor, Dicle University, Faculty of Dentistry, Department of Endodontics, Diyarbakır, TURKEY \\ ${ }^{3}$ Assoc. Professor, Adnan Menderes University, Faculty of Dentistry, Department of Orthodontics, Aydın, TURKEY \\ ${ }^{4}$ Assistant Professor, Mustafa Kemal University, Faculty of Dentistry, Department of Orthodontics, Hatay, TURKEY \\ ${ }^{5}$ Research Assistant, PhD, Dicle University, Faculty of Dentistry, Department of Orthodontics, Diyarbakır, TURKEY
}

\section{Key Words}

Virtual model, cleft lip, cleft palate, CBCT, 3D model

\author{
Correspondence: \\ Torun Ozer, \\ Adnan Menderes University, \\ Faculty of Dentistry, \\ Department of Orthodontics, \\ Aydın, TURKEY \\ e-mail: torunozer@gmail.com
}

\section{Abstract}

Aim: To demonstrate three-dimensional virtual modeling of a cleft lip and palate patient.

Methodology: Traditional two-dimensional treatment planning modalities are often inadequate. Developing technology has enabled the virtual modeling of cleft lip and palate patients. Mimics software was used to demonstrate how to construct such a model.

Conclusion: We are now able to model cleft lip and palate patients in three dimensions.

(Int Dent Res 2012;2(1):43-47)

\section{Introduction}

Cleft lip and palate is a frequent problem seen at birth that can affect the patient for life. Its incidence at birth is not known in some parts of the world, especially in underdeveloped countries. In countries for which information is available, differences in sampling (hospital, population) and inclusion criteria restrict comparability (1). Cleft lip or palate is seen roughly 1 in 700 live births (2). No consistent temporal trend (3) or seasonal pattern (4) has been established.

Cleft lip with or without cleft palate is more frequent in males, whereas isolated cleft palate is more common in females, across various ethnic groups. The sex ratio varies with the severity of the cleft, presence of additional malformations, number of affected siblings in a family, ethnic origin, and, possibly, paternal age $(1,2)$.

No consistent association between orofacial clefts and socioeconomic status has been established (5). However, many of the world's most deprived populations do not have surveillance systems for birth defects, and the perception that the prevalence at birth is high in some of these regions is not evidence based. The World Health Organization International Collaborative Research on Craniofacial Anomalies project is currently addressing gaps in birth defect surveillance, particularly in developing countries.

Non-syndromic orofacial clefts, which include cleft lip, cleft lip and palate, and cleft palate alone, comprise a range of disorders affecting the lips and oral cavity. Effects on speech, hearing, appearance, and cognition can have long-lasting adverse outcomes in terms of health and social integration. Affected children need multidisciplinary care from birth until adulthood and have higher morbidity and mortality throughout life than do unaffected individuals. Studies have shown that many children 
and their families are affected psychologically to some extent (6-8). Although rehabilitation is possible with good care, orofacial clefts inevitably pose a burden to the individual, family, and society, requiring substantial expenditure in terms of health and related services.

Care for children born with these defects generally includes many disciplines: nursing, plastic surgery, maxillofacial surgery, otolaryngology, speech therapy, audiology, psychology, genetics, orthodontics, and dentistry. Furthermore, in both developing and developed countries, standards of care for patients with cleft lip, cleft lip and palate, or cleft palate alone remain a cause for concern (9).

Cleft lip and palate defects not only have a huge impact on the affected individual's life, but also increase healthcare costs, posing a societal burden. Not surprisingly, much research is being conducted to better understand the bones of the skull, to design optimized implants and bone plates, and to improve procedures. Mimics (Materialise, Leuven, Belgium) three-dimensional (3D) modeling software has been introduced commercially for medical image processing (10). Mimics can segment 3D medical images, including computed tomographic (CT), magnetic resonance, cone beam CT (CB-CT), and ultrasound images, to produce a very accurate $3 \mathrm{D}$ model of a patient's anatomy. These patient-specific models can be used for a variety of engineering applications directly in Mimics or 3-matic, or the 3D models and anatomical landmarks can be exported to third-party software, including statistical, computer-aided design, and finite element analysis packages. These programs can be used to produce a stereolithographic model of the patient, which can then be used to produce a prosthesis for a craniofacial defect.

This study outlines the steps used to make a 3D virtual model in a patient with cleft lip and palate to enable practitioners to understand the true extent of the cleft.

\section{Technical Procedure}

After DICOM-formatted data are obtained for the patient to be modeled, they must be imported into Mimics. Mimics may ask the user to determine the patient's top, bottom, left, right, anterior, and posterior sides, which enables the software to show a four-section display of the coronal (upper left), sagittal (bottom left), and axial (upper right) slices, and a blue screen (bottom right; Fig. 1).

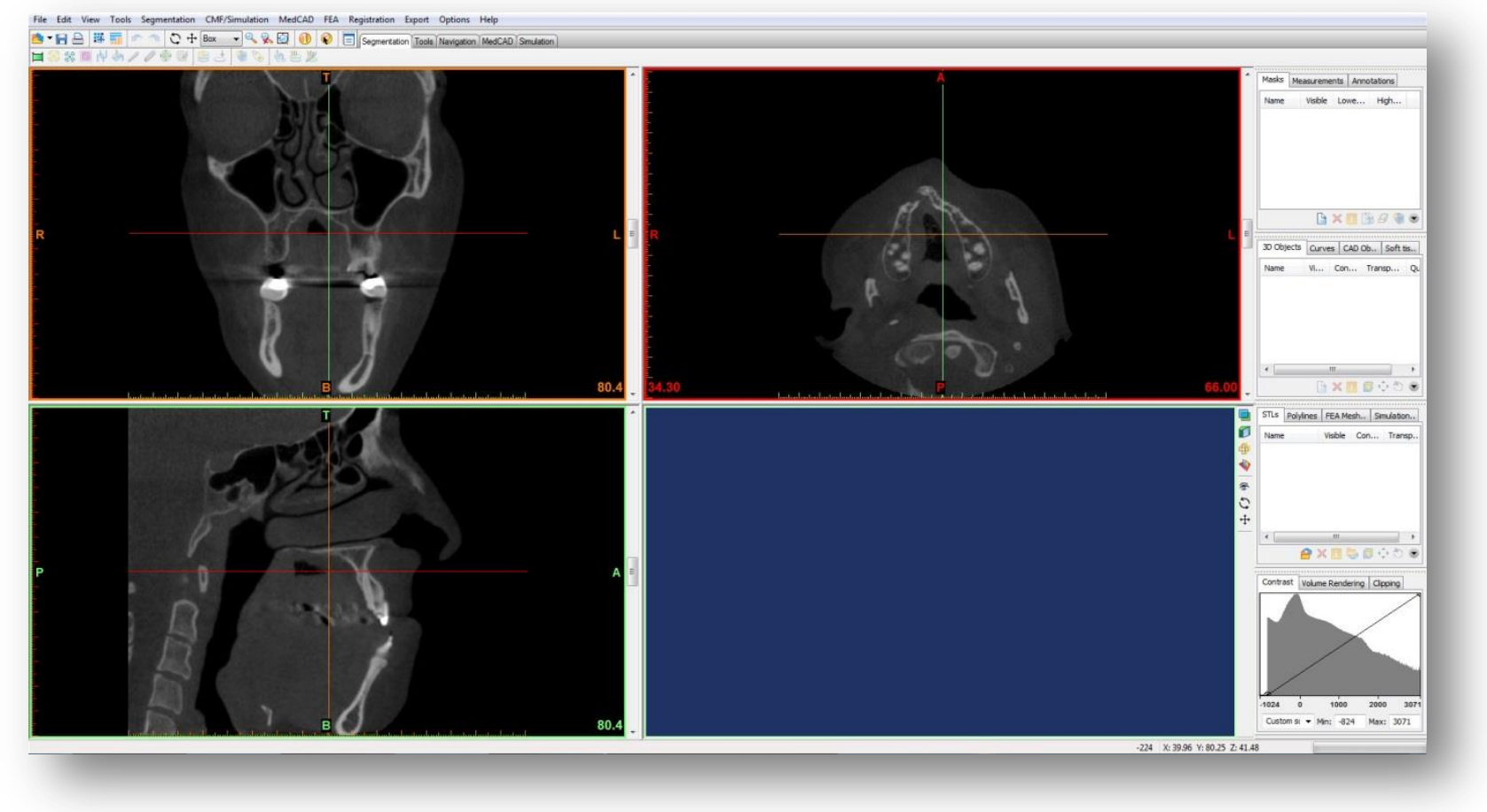

Figure 1: A CBCT slice view of a cleft patient.

Clicking on the "threshold" button on the left side of the tool bar enables the user to choose a custom threshold value or a predefined threshold, such as for bone, soft-tissue, or enamel CT images. 
Here, we chose bone CT values, ( Fig.2) which enable us to demonstrate more features of the software. The three CB-CT slices are painted green. At this time, a mask is made using the "segmentation" tool bar. A small window opens on the screen and clicking the "calculate" button (Fig.
3) generates a green mask in the bottom right window. The mask can be turned, rotated, and zoomed in or out on, by right clicking with the mouse.

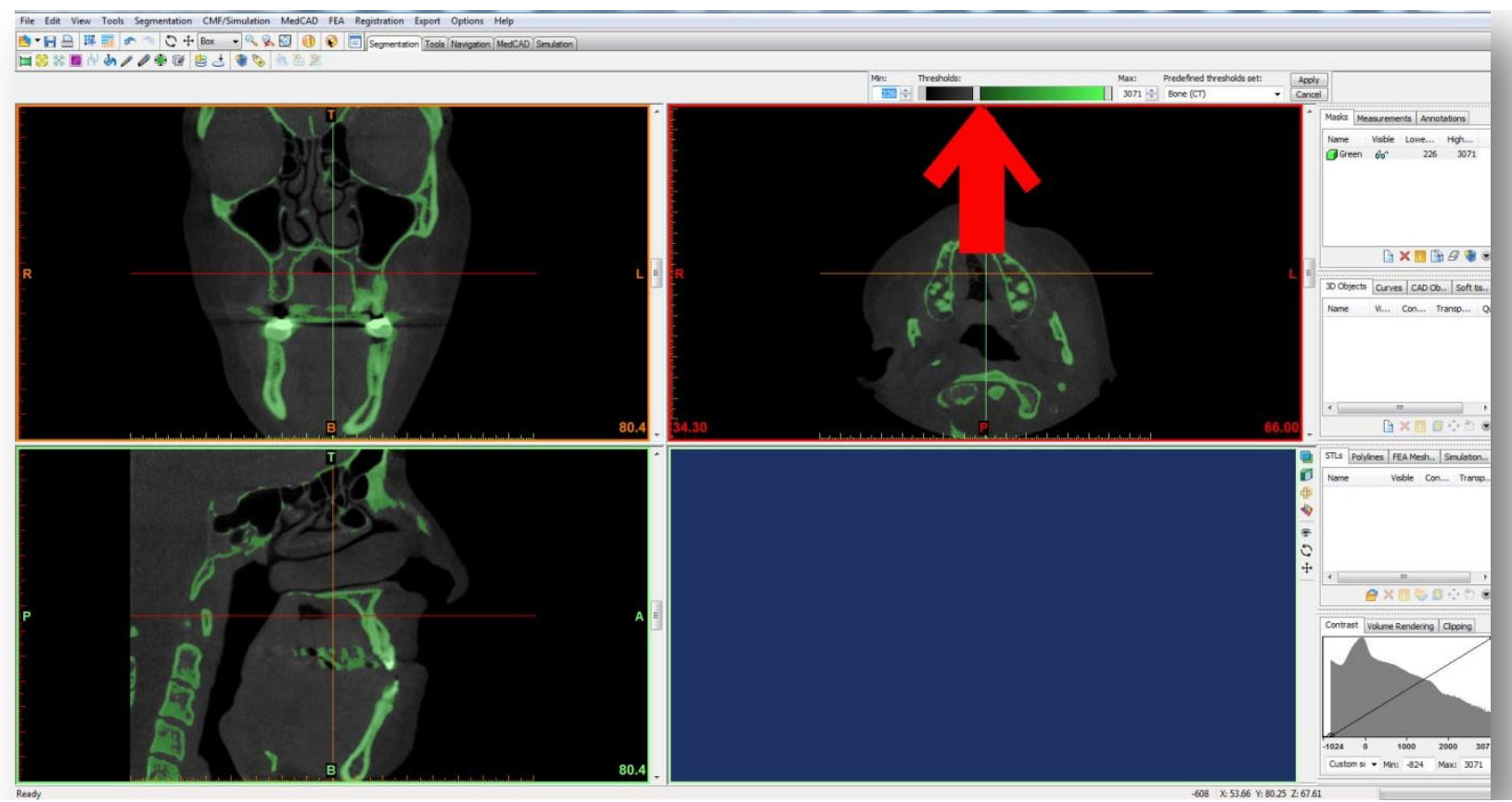

Figure 2: Giving trehshold values.

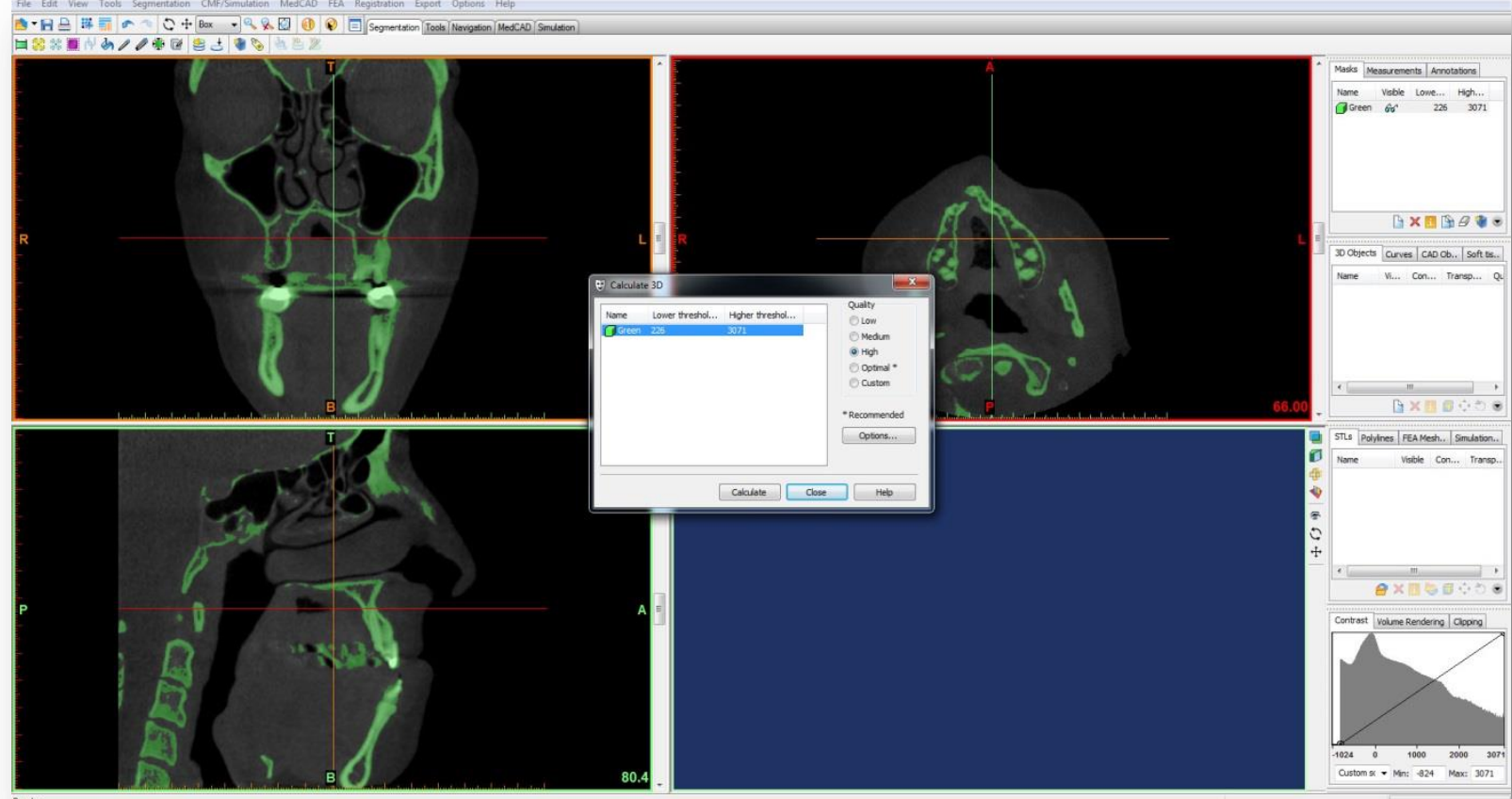

Figure 3: Calculating the three dimensional mask. 
To eliminate unwanted structures that are not in contact with the original mask, one selects the region growing by clicking on a button on the tool bar. From CT slices, the user clicks on the unwanted structures and a new yellow mask appears (Fig. 4). Then, the "calculate $3 \mathrm{D}^{\prime}$ " button is used to generate a new cyan-colored mask, which incorporates both the green and yellow masks. The "Boolean operations" button on the tool bar subtracts the yellow mask from the cyan mask, resulting in a cyan mask of the maxilla, mandible, and cranium. The "Crop mask" button can be used to cut out unwanted structures such as the mandible and cranium, leaving a new mask of the maxilla in the bottom right of the screen. This mask can then be edited from the CT slices. Clicking the "edit masks" button on the tool bar, one must erase the unwanted structures from the axial, coronal, and sagittal views of each slice. Erasing these from the coronal slices, we obtained a 3D virtual model of our cleft lip and palate patient (Fig. 5).

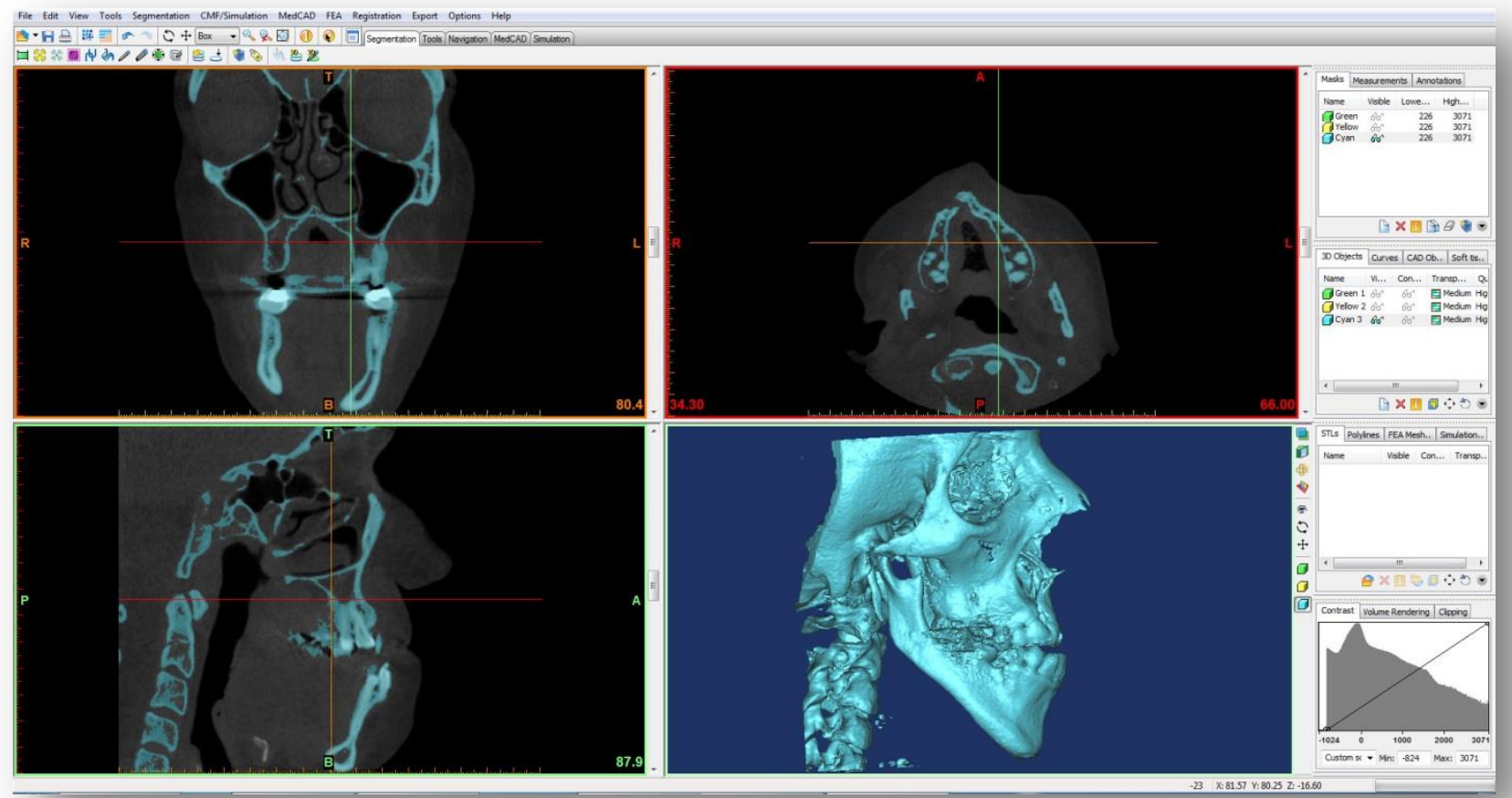

Figure 4: Erasing the unwanted structures that are not in contact with the original mask.

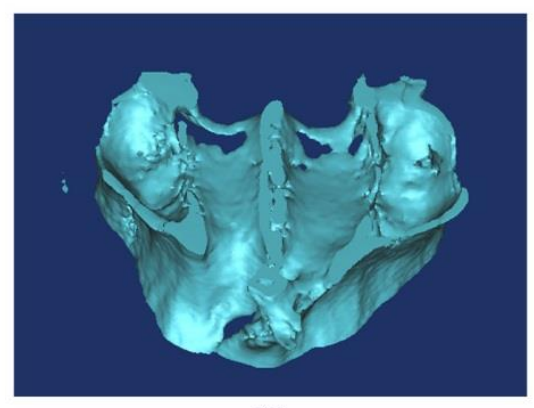

a

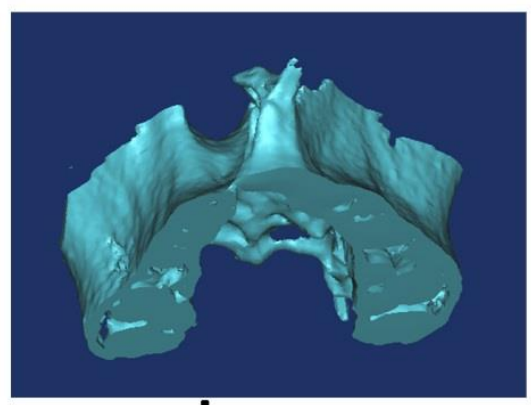

b

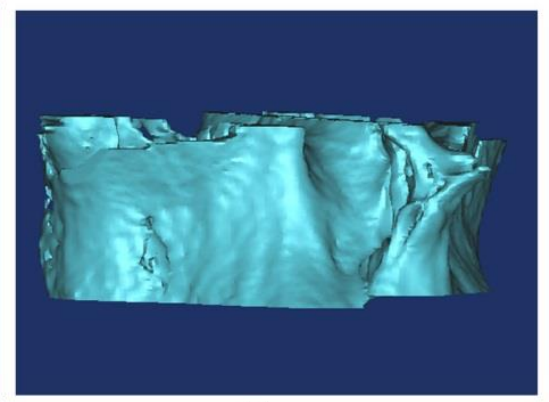

C

Figure 5: A three dimensional model of the maxilla. 


\section{Discussion}

Clefts can also affect the eyes, ears, nose, cheeks, and forehead. In 1976, Paul Tessier (11) described 15 types of craniofacial cleft, most of which are rare. A cleft lip or palate can be treated successfully with surgery, especially when the operation is performed soon after birth or in early childhood. $(7,9)$ The surgery in childhood primarily treats the soft tissues; the underlying bone cannot be seen at this time, preventing clinicians from understanding the true extent of a defect. Different visualization techniques enable us to see the underlying hard tissues.

Osseous defects of the alveolus are present in $75 \%$ of all cleft lip and palate patients. Bone grafting of these defects normalizes facial and dental function. Failure to reconstruct the osseous deformity may result in an oronasal fistula, fluid reflux, speech pathology, anteroposterior or transverse deficiency of the maxilla, lack of bone support for the incisors and cuspids, dental crowding, and facial asymmetry $(8,9)$. Bone grafting unifies the maxilla and is best done after the majority of facial growth is complete and the secondary dentition is erupting. This secondary bone grafting yields the best results (12). Successful bone grafting requires analysis and quantification of the deformity(13), which has traditionally been achieved by direct anthropometry, cephalometry, or visual inspection of a 3D CT model. Before the relatively recent advent of 3D imaging, craniofacial analysis was performed in two dimensions. Therefore, most anthropometric tables and cephalometric norms are two-dimensional. Fortunately, with advances in computer technology, we are now able to perform these analyses in three dimensions.

\section{Conclusion}

The 3D modeling of patients with cleft lip and palate enables us to visualize the defect in more detail and to devise a better surgical treatment plan.

\section{Acknowledgments}

The authors deny any conflicts of interest related to this study. This Project has been supported by Research Fund of the Dicle University. Project Number: 10-DH-95.

\section{References}

1. PA Mossey, J Little. Epidemiology of oral clefts: an international perspective. DF Wyszynski (Ed.), Cleftlip and palate: from origins to treatment, Oxford University Press, New York (2002), 127-58.

2. P Mossey, E Castillia Global registry and database on craniofacial anomalies. World Health Organization, Geneva (2003).

3. International Clearinghouse for Birth Defects Monitoring Systems. Annual report 2001 (with data for 1999) ICBDMS, Rome (2001).

4. EE Castilla, IM Orioli, R Lugarinho et al. Monthly and seasonal variations in the frequency of congenital anomalies Int J Epidemiol, 1990;19:399-404.

5. JD Clark, PA Mossey, L Sharp et al. Socio-economic status and orofacial clefts in Scotland, 1989 to 1998 CleftPalate Craniofac J 2003;40: 481-5.

6. CW Ngai, WL Martin, A Tonks, MP Wyldes, MD Kilby. Are isolated facial cleftlip and palate associated with increased perinatal mortality? A cohort study from the West Midlands Region, 1995-1997 J Matern Fetal Neonatal Med, 2005;17:203-6.

7. K Christensen, K Juel, AM Herskind, JC Murray. Long term follow up study of survival associated with cleftlip and palate at birth. BMJ 2004;328:1405.

8. NW Berk, ML Marazita. The costs of cleftlip and palate: personal and societal implications. DF Wyszynski (Ed.), Cleftlip and palate: from origin to treatment, Oxford University Press, New York (2002), 458-67.

9. WHO Human Genetics Programme. Global strategies to reduce the health-care burden of craniofacial anomalies: report of $\mathrm{WHO}$ meetings on international collaborative research on craniofacial anomaliesWorld Health Organization, Geneva (2002)

10. http//www.materialise.com

11. Tessier P. Anatomical classification of facial, craniofacial and latero-facial clefts. J Maxillofac Surg 1976;4: 69-92.

12. Waite PD, Waite DE. Bone grafting for the alveolar cleft defect. Semin in Orthod 1996;2:192-6.

13. Gateno J, Xia JJ, Teichgraeber JF. New Methods to evaluate craniofacial deformity and to plan surgical correction. Semin Orthod 2011;17:225-34. 